\title{
Host-guest interactions on electrode surfaces for immobilization of molecular catalysts
}

Laurent Sévery ${ }^{1}$, Jacek Szczerbiński ${ }^{2}$, Mert Taskin $^{3}$, Isik Tuncay $^{1}$, Fernanda Brandalise Nunes ${ }^{1}$, Chiara Cignarella ${ }^{1}$, Gabriele Tocci ${ }^{1}$, Olivier Blacque ${ }^{1}$, Jürg Osterwalder ${ }^{3}$, Renato Zenobi ${ }^{2}$, Marcella Iannuzzi ${ }^{1}$ and S. David Tilley ${ }^{1 *}$

${ }^{1}$ Department of Chemistry, University of Zurich, Winterthurerstrasse 190, 8057, Zurich, Switzerland

${ }^{2}$ Laboratory of Organic Chemistry, ETH Zurich, Vladimir-Prelog-Weg 3, 8093, Zurich, Switzerland

${ }^{3}$ Department of Physics, University of Zurich, Winterthurerstrasse 190, 8057, Zurich, Switzerland

Corresponding author email: david.tilley@chem.uzh.ch 


\section{Abstract}

The strategy of anchoring molecular catalysts on electrode surfaces combines the high selectivity and activity of molecular systems with the practicality of heterogeneous systems. The stability of molecular catalysts is, however, far less than that of traditional heterogeneous electrocatalysts, and therefore a method to easily replace anchored molecular catalysts that have degraded could make such electrosynthetic systems more attractive. Here, we apply a non-covalent "click" chemistry approach to reversibly bind molecular electrocatalysts to electrode surfaces via host-guest complexation with surface-anchored cyclodextrins. The host-guest interaction is remarkably strong and allows the flow of electrons between the electrode and the guest catalyst. Electrosynthesis in both organic and aqueous media was demonstrated on metal oxide electrodes, with stability on the order of hours. The catalytic surfaces can be recycled by controlled release of the guest from the host cavities and readsorption of fresh guest. This strategy represents a new approach to practical molecular-based catalytic systems. 
Molecular electrocatalysts can exhibit surprisingly high activities and selectivities that are unmatched by most heterogeneous catalysts. ${ }^{1,2}$ Therefore, the development of robust immobilization strategies for these molecular species on electrode surfaces is of great interest if these molecular catalytic activities and selectivities are to be transferred to more practical heterogeneous electrosynthetic systems, ${ }^{3}$ which have already shown promising results for $\mathrm{CO}_{2}$ reduction, water reduction and water oxidation in the context of storage of renewable energy..$^{4-6}$ Over the past decades, many immobilization strategies have been developed, ${ }^{7}$ which can be categorized into covalent binding (achieved by adding anchoring groups such as carboxylate to the catalysts), ${ }^{8}$ non-covalent binding (pi-stacking on carbon-based electrodes) ${ }^{9,10}$ and polymerization-based binding. ${ }^{11-13}$ Here, we report a new strategy for surface immobilization of molecular electrocatalysts, which relies on a non-covalent "click" chemistry approach to bind molecular species in welldefined sites on electrode surfaces. ${ }^{14}$

The binding of electroactive molecular guests into molecular pockets by means of host-guest complex (HGC) formation has previously been studied by several groups, including those of Stoddart and Kaifer, ${ }^{15}$ Reinhoudt ${ }^{16,17}$ and Huskens. ${ }^{18}$ Liu et al. reported the HGC-formation on gold surfaces with the $\mathrm{C}_{60}$ monoanion as guest, which was found to be electrochemically stable over prolonged durations. ${ }^{19}$ Light-induced electron transfer to and from dye molecules bound via the HGC approach was also demonstrated by Freitag and Galoppini. ${ }^{20,21}$ The group of Sun demonstrated the use of HGC to improve electron transfer between a molecular catalyst and a dye molecule bound onto $\mathrm{TiO}_{2}{ }^{22}$

Among the diverse class of host molecules, cyclodextrins, cucurbiturils and calixarenes are the most studied for HGC formation on different surfaces. ${ }^{23,24}$ For cyclodextrins, the electron transfer rates between the electrode and guest molecules situated inside the surface-bound hosts have been reported to be on the order of $100 \mathrm{~s}^{-1}$, indicating the potential applicability of these systems for molecular electrocatalysis. ${ }^{25}$ It was found that the binding of the guest was not affected by redox processes if the binding units do not undergo oxidation themselves. 
Although rapid electron transfer to HGC-bound molecules has been demonstrated, to the best of our knowledge there have been no reports on using this approach to bind molecular catalysts to electrode surfaces, likely because the dynamic HGC formation in solution phase suggests that rapid desorption would

occur. ${ }^{26}$ We demonstrate, however, that molecular guests bind with surprisingly high stability and show that these are catalytically active. We also show that the binding of the guests is reversible under controlled conditions and exchange of these guests can be induced. We further demonstrate regeneration of the catalytic activity of the electrodes after re-adsorption of fresh electrocatalytic guests, implying the high stability of the host under operational conditions of the bound catalysts.

\section{$\underline{\text { Results and Discussion }}$}

We designed the immobilization system to be as compact as possible, using short tethers to attach the hosts to the electrode surface, ensuring close contact between the electroactive guest and the electrode surface. An aromatic binding unit was chosen as guest binding unit to facilitate electron transfer to the catalytically active site of the guest. With the compact design, displacement of the guests by water or solvent from the opposite face of the host should be inhibited. Higher binding constants of guests in surface-bound hosts compared to the binding in solution have been reported, ${ }^{16,27}$ and the increased binding strength was proposed to be related to further interactions between guest and surface. For hosts with long, flexible chains connecting them to the surface, studies with multi-valent guests showed that HGCs could be stable on a multi-hour time-scale. ${ }^{28}$

\section{Analysis of host-guest complex formation on electrode surfaces}

Following our design criteria, we chose to study the per-thiolated derivate of $\beta$-cyclodextrin, $\mathbf{1}$, on gold as a model for host binding close to the surface (Figure 1 a), as this molecule has been thoroughly studied for its surface-functionalization and surface HGC formation behaviour on gold..$^{15,19,29,30}$ As guests, we chose to investigate ruthenium-based electrocatalyst $\mathbf{2}$, inspired by the class of molecular catalysts that has very 
recently been shown to be active for ammonia oxidation in organic electrolytes (ammonia being a promising candidate as storage medium for hydrogen). ${ }^{31-34}$ We also investigated structurally similar ruthenium-based electrocatalyst 3, a derivate of which we have reported to be active for water oxidation. ${ }^{35}$ As a control, fluorescent platinum complex $\mathbf{4}$ was synthesized, which served as a non-electroactive guest. The complexes used in this study and their crystal structures are given in Figure $1 \mathrm{~b}$-d. The common design feature in the guest metal complexes is the naphthyl substitution on the terpyridine ligand, which was employed as the binding unit of the guests inside the cyclodextrin cavity.

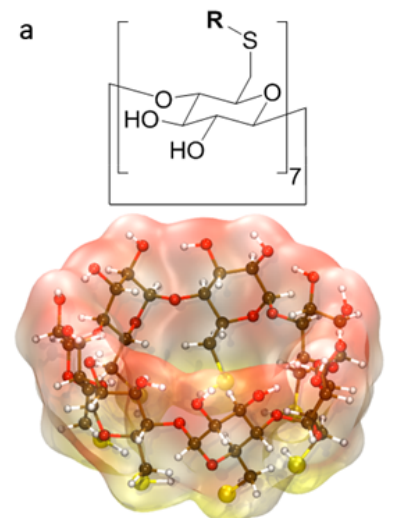

1: $\mathrm{R}=\mathrm{H}$

5: $\mathbf{R}={ }_{\mathrm{PO}_{3} \mathrm{H}_{2}}^{\mathrm{PO}_{3} \mathrm{H}_{2}}$ b
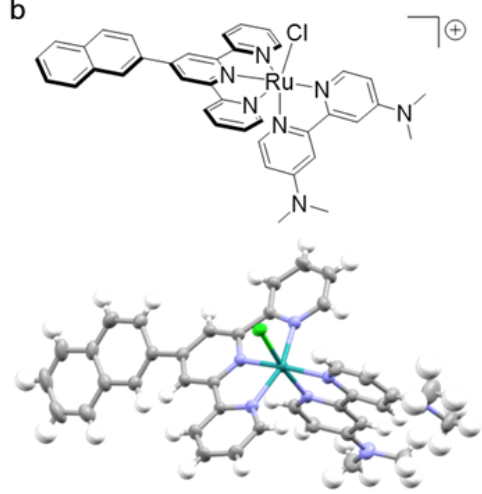

2
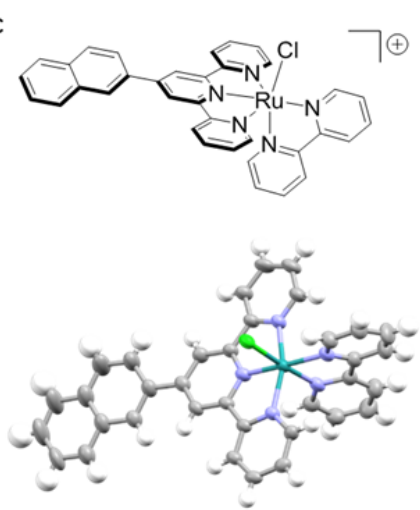

3

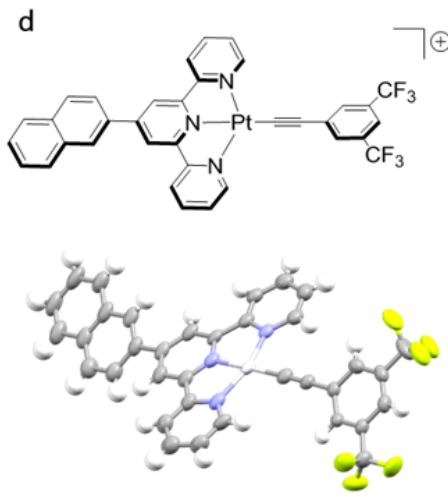

4

Figure 1. Structures of the main compounds used in this paper: a) host $\mathbf{1}$ (thiolated cyclodextrin) and host $\mathbf{5}$

(bisphosphonated cyclodextrin), along with a depiction of the host cavity; b) guest $\mathbf{2}$ c) guest $\mathbf{3}$ d) guest $\mathbf{4}$. The crystal structures of guests are displayed showing the $50 \%$ thermal ellipsoids. Solvent and counterions $\left(\mathrm{PF}_{6}{ }^{-}\right.$for guests 2 and 3 ; $\mathrm{ClO}_{4}^{-}$for guest 4) are omitted for clarity.

To confirm that binding between the guests and cyclodextrin takes place in solution, fluorescence titrations and NMR experiments were performed (see Figures S1 and S2 in Supporting Info). A 1:1 stoichiometry binding constant for fluorescent guest 4 with $\beta-C D$ was determined and a binding constant of $\mathrm{K}_{11}=6.5 \cdot 10^{3} \mathrm{M}^{-1}$ was obtained, which is 2-4 times higher than for other naphthyl-based guests with $\beta-C D .{ }^{36,37}$ 
The attachment of 1 on gold substrate was investigated by X-ray photoelectron spectroscopy (XPS) (Figure S3) and tip-enhanced Raman spectroscopy (TERS) (vide infra). The spectra indicated the expected attachment of the cyclodextrin host via the thiol groups, as was evidenced by the loss of the $v(\mathrm{~S}-\mathrm{H})$ Raman band upon adsorption. The surface density of 1 on Au(111) determined by XPS was found to be up to $0.46 \mathrm{~nm}^{-2}$, corresponding to a relatively compact layer of host molecules on the substrate. We note that the maximum coverage of the surface was achieved with dipping times of 3 minutes, with no further increase in the surface coverage of 1 at longer exposure of the gold substrate (Figure S4).

The formation of HGCs with the gold-bound 1 was investigated using TERS, and DFT calculations were performed to gain a better understanding of this system. ${ }^{38}$ The optimization of 1 on an $\mathrm{Au}(111)$ slab (more details in Methods and SI) revealed the expected binding of the host via all seven thiol groups, despite the mismatch in symmetry with the gold substrate (Figure 2a), and is consistent with the complete loss of the $v(\mathrm{~S}-\mathrm{H})$ band in the TER spectra of 1 on Au (see Figure 3a). 


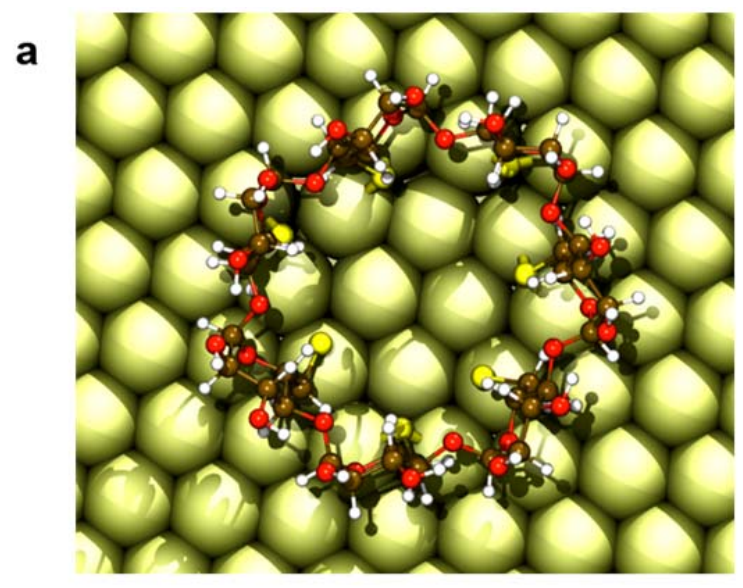

b
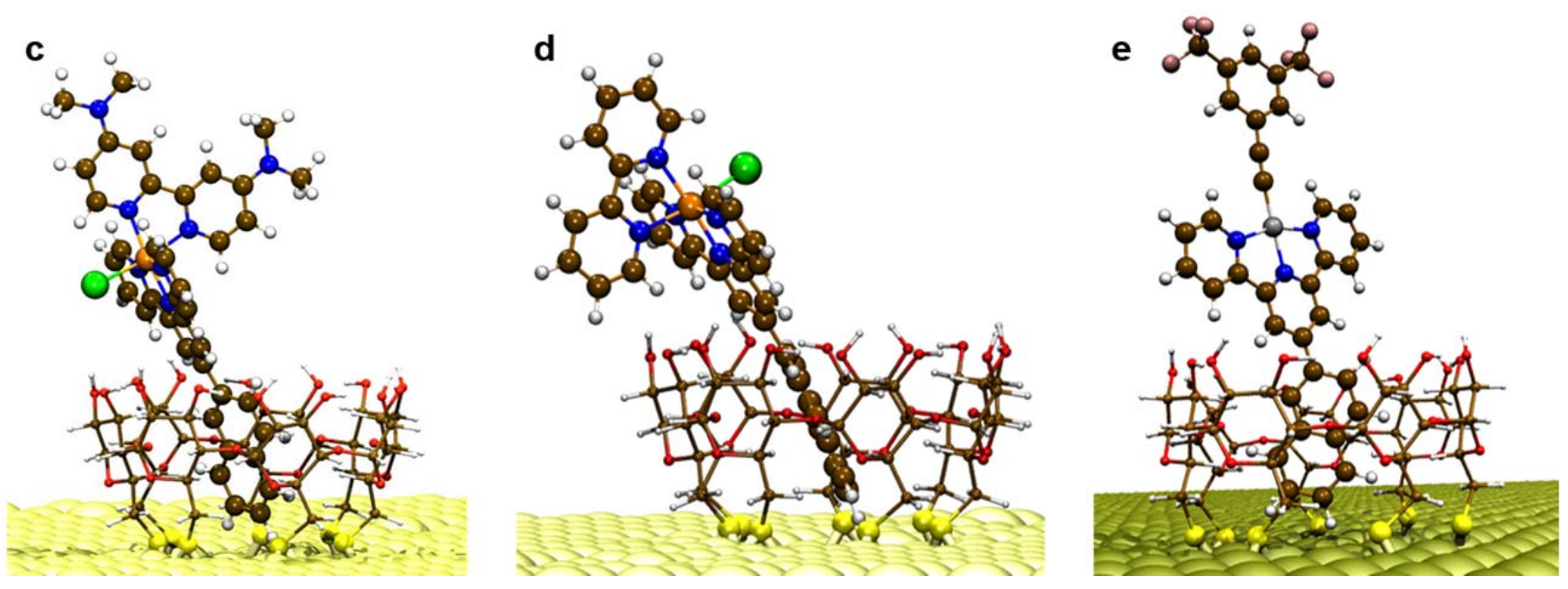

Figure 2. DFT optimized binding structure of 1 on gold in a) plan view and b) side view, showing each thiol group bound to gold in a unique binding geometry. c), d) and e) The optimized structures of HGC bound metal guests 2, 3 and 4, respectively, on gold show the guests bound tightly into the host cavity very close to the surface of the electrode, with the catalytically active site (for guests 2 and 3) above the top face of the host.

Formation of the HGCs was first computed at the DFT level of theory for all guests in the gas phase (more details in SI and Figures S5, S6 and S7), which gave binding energies between 1 host and the guests of -2.03 $\mathrm{eV}$ (guest 4) and -2.17 eV (guests 2 and 3). On the Au(111) surface, the binding energies between guests and surface-bound 1 were found to be between $-4.31 \mathrm{eV}$ and $-4.89 \mathrm{eV}$, which reveals a dramatic increase in adsorption energy into the cyclodextrin cavity. This large increase in the adsorption energy is related to delocalization of electronic states of the guest molecules and the gold substrate (see Figure S8 and S9), which 
is evidenced by significant mixing of gold and guest orbitals. The strong interactions and delocalization of electronic states indicate that oxidation or reduction of electroactive guests ( $\mathbf{2}$ and $\mathbf{3}$ ) can be easily achieved. However, strong coupling also implies that the diagnostic redox peaks of the guest in cyclic voltammetry (CV) experiments would be absent, similar to the case with graphite-conjugated molecules that lack clear redox peaks in CV experiments. ${ }^{39}$ Indeed, we did not observe redox peaks for the ruthenium-based guests bound to 1 on gold at the respective redox potentials found in solution (Figure S10), even as a low catalytic current was observed from the HGC of guest $\mathbf{2}$ with host 1 on dodecanethiol-poisoned gold substrates (Figure S11).

The large binding energies obtained from the DFT calculations provide an understanding of the experimentally observed greater stability of the guests bound to the host-functionalized surface versus HGC binding in solution. Thus, conditions need to be developed to actively remove the guests from the surfacebound hosts for recycling purposes. The desorption of guests under competitive HGC formation in solution has been reported, ${ }^{28,40}$ and this strategy was also employed to show that guest binding to the $\mathbf{1}$ modified gold substrates could be controlled under specific conditions.

Using TERS (Figure 3a), we were able to track the presence of guest $\mathbf{3}$ on gold functionalized with host 1. Formation of the HGC on the Au surfaces was visualized by the appearance of an intense Raman fingerprint of the guest between 1000 and $1600 \mathrm{~cm}^{-1}$ compared to the moderately strong $\mathrm{v}(\mathrm{C}-\mathrm{H})$ band of 1 at $2900 \mathrm{~cm}^{-1}$ (cyclodextrins are very weak Raman scatterers). Desorption of the majority of guest was achieved by soaking and sonication of the sample in a concentrated $\beta-C D$ solution (indicated by the strong decrease in the guest/host Raman band intensity ratio). Re-adsorption of the guest was observed after re-soaking the desorbed sample in a solution of 3. In a separate experiment, we also showed that guest 4 (Pt complex) can be exchanged for a second guest $\mathbf{2}$ by initial desorption of $\mathbf{4}$ using concentrated $\beta-C D$ solution and subsequent incubation in a solution of guest $\mathbf{2}$ (Figure 3b). The exchange was enabled by the release of guest $\mathbf{4}$ from the surface-bound host cavities during the desorption step and cannot be explained with stronger binding of guest $\mathbf{2}$ compared to guest $\mathbf{4}$, as the reverse exchange ( $\mathbf{2}$ for $\mathbf{4}$ ) is also feasible (Figure S12). Interestingly, 
complete loss of guest signal after the desorption treatment was never observed, which again hints towards the unexpectedly strong interaction between the guest and the 1-functionalized gold. Raman and TERS measurements of guest 2 revealed a red-shift of the guest's optical absorption maximum upon host-guest binding on the gold surface, which illustrates the shrinking of the HOMO-LUMO gap predicted by the DFT calculations, providing further evidence for the strong coupling between the gold substrate and the guest (Figure S13). Direct observation of 1 and the HGCs on Au(111) was attempted by STM, however a clear image could not be obtained despite significant efforts (Figure S14).

Studying the electrochemical properties of the HGCs on gold alone was not sufficient to obtain a clear picture of our catalytic system. Gold electrodes are known to undergo surface oxidation, ${ }^{41}$ which leads to the desorption of thiol-based adsorbates. ${ }^{42,43}$ Furthermore, the relatively low surface area and high background activities of gold for many catalytic processes make the analysis of catalytically active adsorbates very difficult. $^{44}$

To address these issues and to compliment the array of available analytical techniques to study the surfacebound HGCs, metal oxide (MO) substrates were used, specifically indium tin oxide (ITO), zirconium dioxide $\left(\mathrm{ZrO}_{2}\right)$, and titanium dioxide $\left(\mathrm{TiO}_{2}\right)$. As these materials have similar properties in terms of surface-adsorption chemistry, ${ }^{45}$ the choice of substrate can be tailored to the application of analytical technique (ITO for electrochemical measurements, $\mathrm{ZrO}_{2}$ for fluorescence and IR, and $\mathrm{TiO}_{2}$ for solid state NMR). Furthermore, by preparing mesoporous layers of the MOs, higher surface areas are easily accessible, allowing for the use of further spectroscopic methods and simplifying the analysis of electrochemical data. 
a
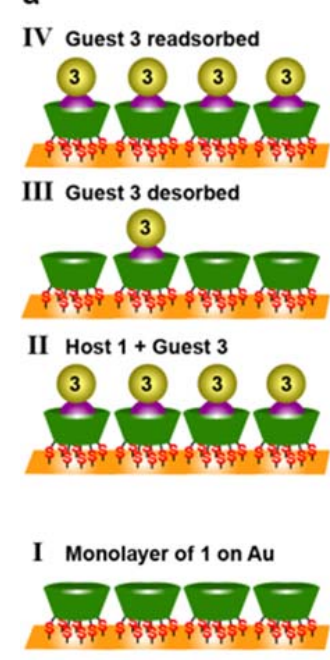

Powder 1 (bulk Raman)

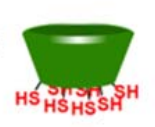

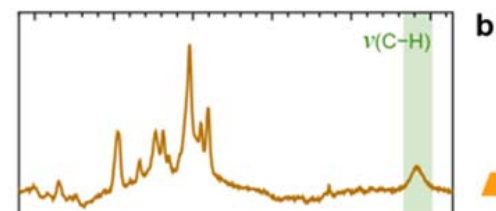
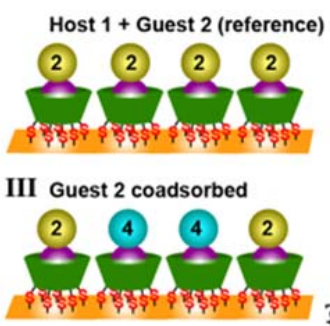

II Guest 4 desorbed

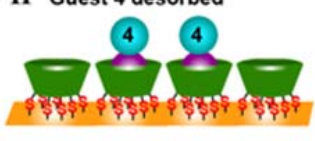

I Host 1 + Guest 4

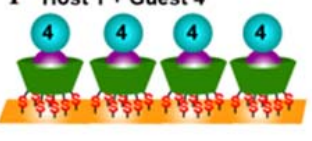

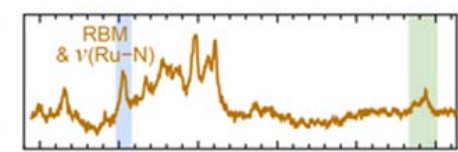

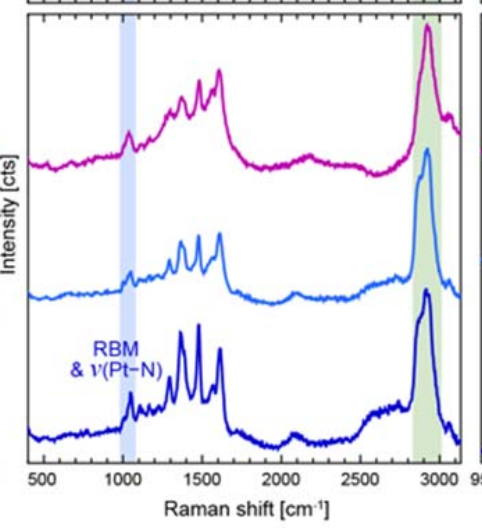

950100010501100 Raman shift $\left[\mathrm{cm}^{-1}\right]$

Figure 3. Regeneration and exchange of the catalysts on the host-functionalized Au surface. a) The binding of 1 to Au via all seven thiolate groups is confirmed by the complete absence of the S-H stretching vibration in the TERS spectrum I of 1-functionalized gold. Reversible adsorption and desorption of $\mathbf{3}$ on host-functionalized gold is reflected in the varying intensity of the peaks at $1000-1600 \mathrm{~cm}^{-1}$ in spectra II-IV. The spectra are normalized with respect to the C-H stretching band of 1 at $2900 \mathrm{~cm}^{-1}$ (highlighted in green). b) Desorption and subsequent exchange of 4 by 2 on host-functionalized gold is confirmed by the shift of the ring breathing modes (RBM) of the metal-coordinated pyridyl rings from $1050 \mathrm{~cm}^{-1}$ for Pt toward $1030 \mathrm{~cm}^{-1}$ for Ru (highlighted in blue). The incomplete desorption of $\mathbf{4}$ is reflected in the residual intensity of 4 in spectrum II and in the mixed signals of $\mathbf{4}$ and $\mathbf{2}$ in spectrum III.

To transfer the HGC chemistry from the gold to the MO substrates, two strategies were employed: direct introduction of multiple suitable binding groups, geminal bisphosphonates (BP), ${ }^{46}$ to the host scaffold (host $\mathbf{5}$, see Figure 1) or pre-functionalization of the MO substrates with propiolic acid and subsequent thiol-yne click chemistry on the surface. ${ }^{47}$ Both strategies are schematically shown in Figure S15. The host molecules on MO substrates are slightly further away from the surface, and electrons are more localized and tightly bound in MOs. ${ }^{48,49}$ These factors may lead to decreased electronic coupling of the guests with the surface. 
Surface binding of the CD hosts was analysed by IR spectroscopy, XPS and solid state NMR (Figures S16, S17 and S18 in SI). Furthermore, impedance spectroscopy revealed the increased charge-transfer resistance after binding of host 5 to an ITO electrode, which is in accordance with previous observations on gold electrodes

(Figure S19). ${ }^{29}$ Submersion of the host-functionalized electrodes into guest solutions led to the formation of surface-bound HGCs accompanied by physisorbed guest species. The presence of the guests on the surface could be clearly determined by various spectroscopic methods and by electrochemical measurements (Figures S20, S21, S22 and S23). Removal of physisorbed species was achieved by treatment of the samples with $100 \mathrm{mM}$ acetic acid in methanol, as was determined by the loss of guest fluorescence signal from a physisorbed sample on $\mathrm{ZrO}_{2}$ (Figure S24)). Formation of the host-guest complexes could also be seen during cyclic voltammetry experiments: whereas physisorbed guests rapidly desorb after a few cycles, redoxfeatures of the HGCs could be observed even after extended cycling (Figure S25). A binding constant for HGC formation of guest 3 with host 5 on $\mathrm{ZrO}_{2}$ of $\mathrm{K}_{11}^{\text {surf }}=3.3 \cdot 10^{5} \mathrm{M}^{-1}$ was calculated based on a Langmuir-type model (Figure S26).

Exposing mesoporous $\mathrm{ZrO}_{2}$ substrates with $\mathrm{HGC}$-bound guests to the desorption conditions used for the gold (with heating of the solution instead of sonication, to preserve the mesoporous substrate), the removal of guests from the surface could be tracked by fluorescence spectroscopy. The same reversible desorption and re-adsorption of different guests (as was shown for the gold electrode using TERS) indicates that the surface can be modified without degrading the surface-bound hosts (Figure S27).

\section{Catalysis using host-guest complex bound molecular electrocatalysts}

Catalyst 2 -designed as a molecular ammonia oxidation catalyst and equipped with a naphthyl binding groupshows the expected homogeneous phase catalytic activity for $\mathrm{NH}_{3}$ oxidation in THF solution around its first oxidation potential (Figure 4a). A sample with HGC-bound $\mathbf{2}$ was then tested under the same conditions and also showed a clear onset of catalysis at the same potential as in solution (around $-0.1 \mathrm{~V} \mathrm{vs}$. Fc/Fc ${ }^{+}$, Figure $4 \mathrm{~b}$ ). The control samples with non-active guest 4 showed a much later onset of $\mathrm{NH}_{3}$ oxidation (taking place 
directly on the electrode surface), and physisorbed catalyst led to lower current with a rapid loss of activity during operation (Figure S28).

\section{Homogeneous}

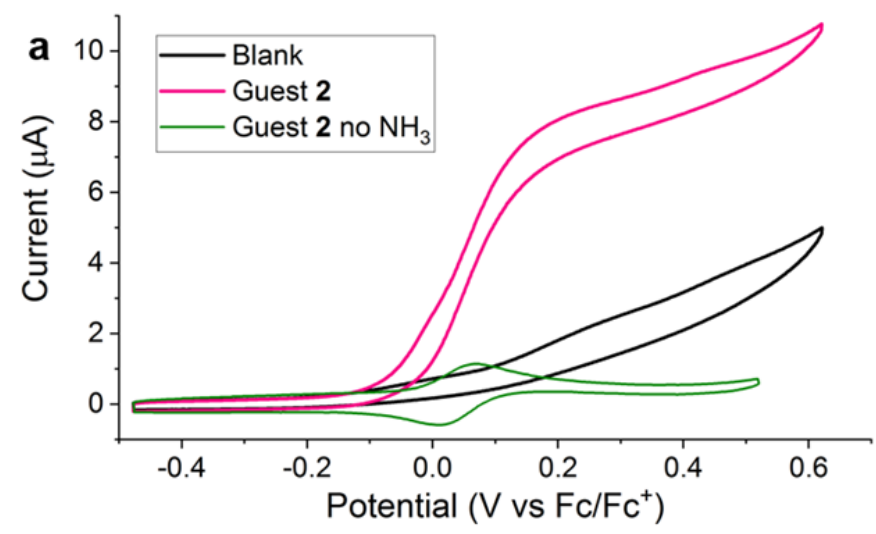

Heterogeneous

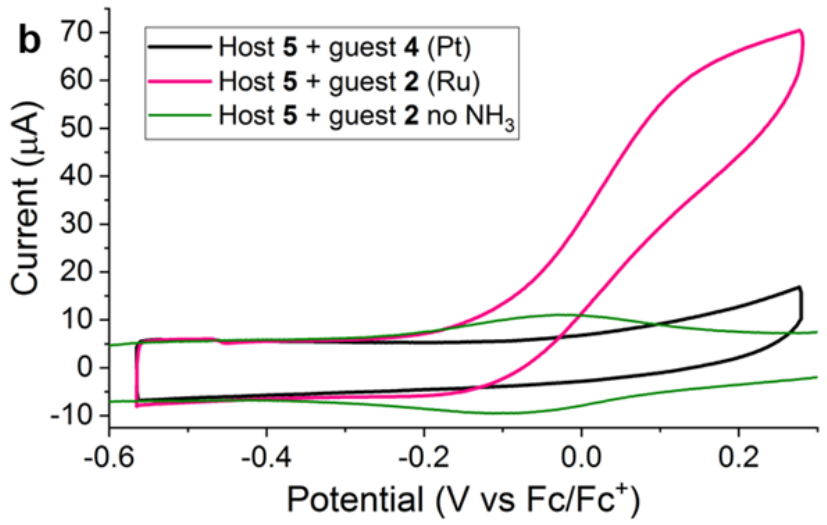

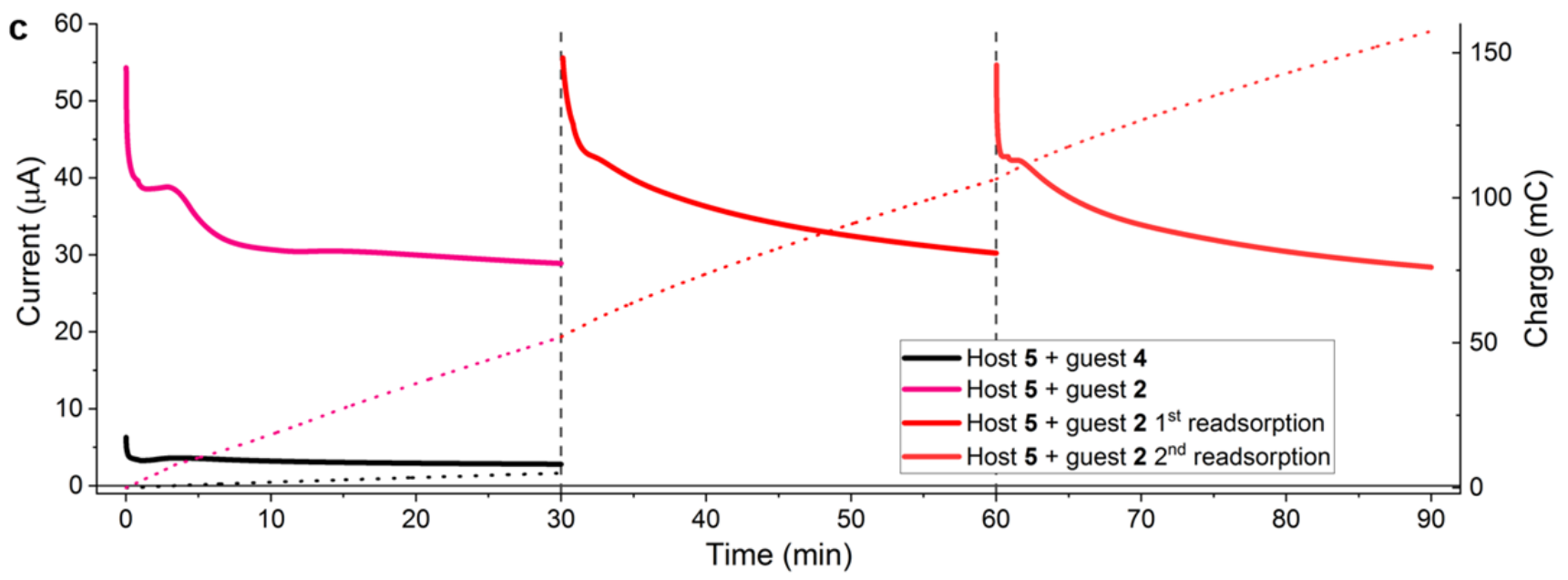

Figure 4. Electrocatalytic oxidation of $\mathrm{NH}_{3}$ in THF solution. a) $\mathrm{CV}$ scan under homogeneous reaction conditions using an ITO working electrode in $\mathrm{NH}_{3}$ saturated THF solution (0.2 M TBAPF 6 and $0.1 \mathrm{MNH}_{4} \mathrm{PF}_{6}$ as conducting salt and proton source; $10 \mathrm{mV} / \mathrm{s}$ scan rate). Without catalyst (black) shows low activity. For a $0.1 \mathrm{mM}$ solution of guest 2 in the absence of $\mathrm{NH}_{3}$, the $\mathrm{Ru}(\mathrm{II}) / \mathrm{Ru}(\mathrm{III})$ redox peak is observed (green). Upon addition of ammonia, a catalytic onset for ammonia oxidation is observed at the Ru(II)/Ru(III) oxidation potential (magenta). b) CV scans of HGC-bound guests 2 (magenta) and 4 (black) with host 5 on mesoporous ITO show the same catalytic behaviour in the presence of the ruthenium catalyst, with onset at the $\mathrm{Ru}(\mathrm{II}) / \mathrm{Ru}(\mathrm{III})$ oxidation potential measured for $\mathrm{HGC}$-bound 2 without $\mathrm{NH}_{3}$ (green). The

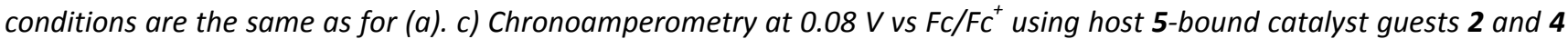


(the latter as a non-catalytically active reference). Readsorption of guest $\mathbf{2}$ after 30 minutes of catalysis into the hostfunctionalized electrodes leads to complete regeneration of catalytic activity.

The stability of the HGC-bound electrocatalyst $\mathbf{2}$ under operation was analysed by chronoamperometry, revealing a slow decrease in current to roughly $70 \%$ of the initial value over 30 minutes (Figure 4c), with significantly higher currents observed than for physisorbed $\mathbf{2}$ without host present (Figure S29). Readsorption of fresh $\mathbf{2}$ regenerated the catalytic activity of the electrode, which clearly indicates that the host molecules on the electrode surface are intact and can be used to bind fresh catalyst guests. The decrease of current could either be due to slow desorption of the guests or degradation of the HGC-bound catalyst. Nevertheless, these results show that our immobilization strategy of molecular electrocatalysts using HGCs can be employed to bind active catalysts to electrodes. Moreover, this strategy was effectively used in organic electrolyte, which is notable considering the general competition for occupying the host cavity between guests and solvent molecules. ${ }^{50,51}$ This observation underlines the strong adsorption interaction between the guest molecules and the surface bound hosts. Additional soaking experiments of the HGC in organic solvents used in electrochemical catalysis revealed hours of HGC stability in methanol, acetonitrile, dichloromethane and toluene, indicating that a wide range of electrocatalytic conditions are compatible with this approach (Figures S30 and S31).

Electrochemical water oxidation was also attempted using guest 3, which was shown to be active (though sluggish) for this reaction in solution (Figure S32a). Unfortunately, we could not observe an unambiguous catalytic response for water oxidation versus the background. To nevertheless confirm the activity of HGCbound guests, we were able to demonstrate catalytic oxidation of a water-soluble phosphine to the corresponding phosphine oxide with HGC-bound $\mathbf{3}$ on ITO as a model reaction (Figure S33). This system also showed similar stability as the case for $\mathrm{NH}_{3}$ oxidation in organic media. Regeneration of catalytic activity upon readsorption of $\mathbf{3}$ indicates the robustness of the host on the surface (Figure S34). 
To confirm that the host is stable during electrocatalysis, we analysed host $\mathbf{5}$-functionalized ITO substrates before and after electrochemical measurements (with HGC-bound guest 2). We observed no peak shifts or decreased intensities for the carbon, phosphorus or sulfur signals pertaining to host $\mathbf{5}$ between the two measurements (Figure 5) indicating that there is no oxidation on any part of the host structure. To demonstrate the stability also under challenging conditions for most classically anchored catalysts, CV cycling at $\mathrm{pH} 10$ under strong oxidative bias was performed (Figure S35), showing only a slow decrease of the guest redox features. Analysis of the sample surface by XPS showed a modest decrease of carbon, sulfur and phosphorus related to surface-bound 5 (Fig S36), indicating that the majority of the surface binding sites remained stable under such harsh conditions on the order of hours. The stability of host $\mathbf{5}$ under catalytic conditions implies the potential use of this immobilization strategy for different molecular electrocatalysts, with the option of electrode recycling. This also implies that the stability of the anchoring group for surface binding can be decoupled from catalyst design and separately optimized on the host, which presents a promising new approach to developing and improving new ways of coupling molecular electrocatalysts with different electrode surfaces. 
a

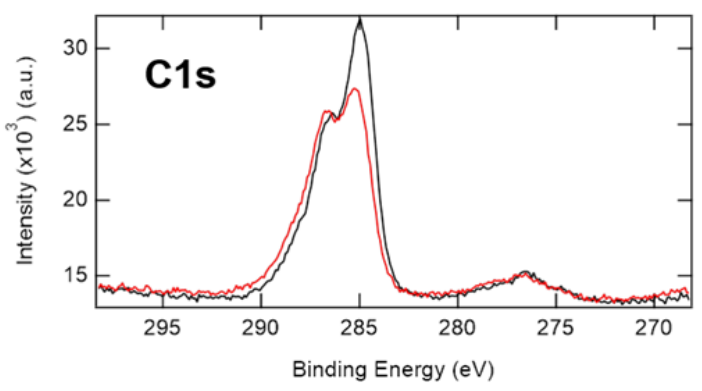

C

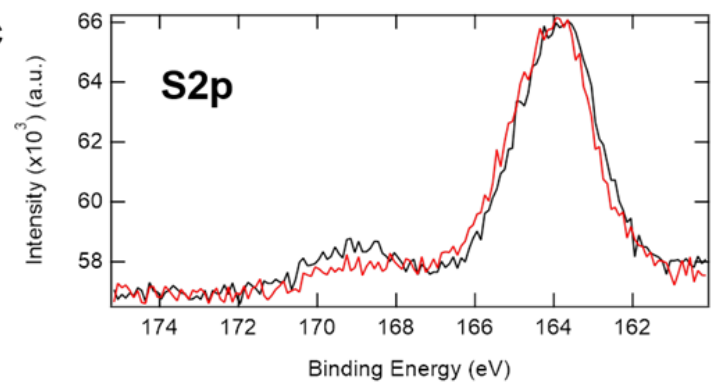

b

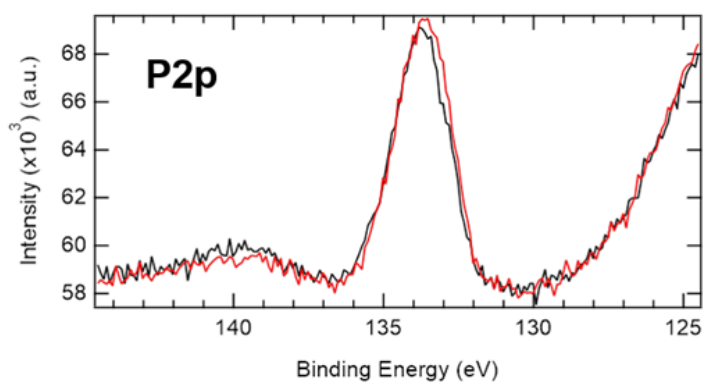

Host 5+Guest 2 Host 5+Guest 2 after e-chem

Figure 5. XP spectra of ITO electrodes functionalized with host $\mathbf{5}$ and HGC-bound guest $\mathbf{2}$ before and after electrocatalytic measurements ( $\mathrm{NH}_{3}$ oxidation in THF solution). a) C1s spectra. b) P2p spectra. c) S2p spectra. For S2p and P2p, no difference between the sample before and after electrocatalysis is observed, showing no change in oxidation state or decrease in surface coverage for either of the elements. In the case of $\mathrm{C} 1 \mathrm{~s}$, a decrease in signal intensity is only observed for the peak at $285 \mathrm{eV}$ which is attributed to adventitious carbon, with the signal from host C-O carbon species remains unchanged. Overall the data indicate that no degradation of the host takes place under operating conditions.

\section{Conclusion}

Host-guest complex formation on electrode surfaces can be used to immobilize molecular electrocatalysts. A detailed study on the formation of such HGCs on gold, along with DFT calculations, shows a strong binding of the guests to the surface-bound cyclodextrin hosts. By demonstrating the catalytic activity of HGC-bound guests in both organic and aqueous electrolyte, our experiments show the versatility of using this approach to immobilize different electrocatalytic guests for operation in different chemical environments. The high stability of the host structures on the electrode surface allows for regeneration of the electrodes by 
re-adsorption of fresh catalyst guests, which overcomes one of the largest challenges in immobilized molecular electrocatalysis: the electrodes can be reused even when the catalyst molecules have degraded.

Our work sets the basis for the use of HGC chemistry for catalyst immobilization. Future research efforts focusing on the preparation of new host structures and families, expanding the scope of the catalytic reactions and understanding and improving the control over the interactions between guest, host and surface will allow for this immobilization strategy to be used for many different applications.

\section{Methods}

Host-functionalization of gold substrates: Gold substrates were used either as prepared (directly after deposition of the gold layer) or cleaned in an $\mathrm{O}_{2}$ plasma before functionalization. Compound $\mathbf{1}$ was attached to the gold by soaking the substrates in a solution of $1(0.1 \mathrm{mM})$ prepared with either DMSO or DMF. Although adsorption was found to take place in less than 5 minutes, the substrates were soaked for $1 \mathrm{~h}$. The substrates were then soaked in pure solvent ( 5 minutes), $\mathrm{MeOH}\left(5 \mathrm{~min}\right.$ ) and dried under a stream of $\mathrm{N}_{2}$. The host-functionalized gold was used immediately or dipped in guest solutions very soon after preparation.

\section{Host-functionalization of metal oxide substrates}

Two-step procedure for binding of host 1: The metal oxide substrate was dipped in a solution of propiolic acid $(10 \mu \mathrm{l}$ in $10 \mathrm{ml} \mathrm{MeCN})$ for $30 \mathrm{~min}$. After this, the substrates were dipped in pure MeCN for 5 min to remove excess propiolic acid, then dried under a stream of $\mathrm{N}_{2}$. The samples were subsequently immersed in a solution of $1(0.7 \mathrm{mM})$ in DMF $(10 \mathrm{ml})$ containing $\mathrm{NEt}_{3}(20 \mu \mathrm{l})$ for $1 \mathrm{~h}$. The substrates were then soaked in pure DMF (15 ml, $5 \mathrm{~min})$, pure $\mathrm{MeOH}(10 \mathrm{ml}, 5 \mathrm{~min})$ and finally dried under a stream of $\mathrm{N}_{2}$.

Direct binding procedure for host 5: The salt form of 5 was dissolved in $\mathrm{H}_{2} \mathrm{O}$ to give a $0.1 \mathrm{mM}$ solution, to which was added $1 \mathrm{v} \%$ of $1 \mathrm{M} \mathrm{H}_{2} \mathrm{SO}_{4}$, resulting in a $\mathrm{pH} 2$ solution. The metal oxide substrate was then 
immersed in this solution for $1 \mathrm{~h}$, after which it was placed in pure $\mathrm{H}_{2} \mathrm{O}(15 \mathrm{ml}, 5 \mathrm{~min})$ and $\mathrm{MeOH}(10 \mathrm{ml}, 5$ min). The samples were then dried under a stream of $\mathrm{N}_{2}$.

Guest functionalization of substrates: The appropriate substrates were immersed in a solution of the guest in $\mathrm{MeOH}(0.05-0.1 \mathrm{mM}$ ) for $1 \mathrm{~h}$, after which they were soaked in pure $\mathrm{MeOH}$ twice ( 5 minutes each). The substrates were then dried under a stream of $\mathrm{N}_{2}$. HGC formation was easily observed by eye on mesoporous substrates due to coloration of the films.

Tip-enhanced Raman spectroscopy: TERS measurements were performed on the host-functionalized gold samples using electrochemically etched silver tips and a $632.8 \mathrm{~nm}$ He-Ne laser. The samples were placed into a scanning tunneling microscope equipped with a $0.7 \mathrm{NA}$ top-illumination objective. The laser beam was focused on the tip-sample junction, and plasmon-enhanced Raman spectra were collected from the molecules residing in the plasmonic hot spot under the tip. The spectra in Figures 3, S12 and S13 are average spectra collected over grids of 100-225 locations. This approach, along with the low laser power applied (1.4$21.2 \mathrm{~kW} / \mathrm{cm}^{2}$ ), allowed the minimization of photoinduced sample damage.

DFT calculations: Optimized structures, energies and electronic properties were obtained by DFT simulations using the Gaussian and plane waves method as implemented in the CP2K program package ${ }^{38}$ The HGCs on gold were simulated using an $\mathrm{Au}(111)$ slab model with four 9x9 layers and periodic boundary conditions were applied. The adsorption energies of the host-guest complexes in the gas phase were computed by comparing the energy of the optimized complex and the energies of the individually optimized CD and catalyst $E_{\text {Ads }}=E_{H G, \text { gas }}-\left(E_{\text {host,gas }}+E_{\text {guest,gas }}\right)$. The adsorption energy of the guest into the anchored CD on $\mathrm{Au}(111)$ were computer as $E_{A d s}=E_{H G, A u}-\left(E_{\text {host }, A u}+E_{\text {guest, gas }}\right)$. 


\section{Acknowledgements}

S.D.T. thanks the University of Zurich, the University Research Priority Program LightChEC, and the Swiss National Science Foundation (PYAPP2 160586) for funding. J.O. also acknowledges funding from the LightChEC. The authors would like to thank Dr. Thomas Fox for the measurements of the solid state NMR spectra. Dr. Thomas Moehl is thanked for assistance and fitting of the impedance data.

\section{Author Contributions}

L.S. and S.D.T conceived and the project. L.S. performed synthesis, electrochemical and catalytic experiments. I.T. assisted with synthesis and electrochemical experiments. J.S. and R.Z. conducted and evaluated TERS experiments. M.T. and J.O. conducted and evaluated XPS and STM experiments. O.B. measured and refined crystal structures. G.T., C.C., F.B.N and M.I. designed, conducted and evaluated calculations. L.S. and S.D.T wrote the manuscript. All authors contributed to discuss the results and revise the manuscript.

\section{Competing Interests}

The authors declare no competing interest. 


\section{References}

1. Brazzolotto, D. et al. Nickel-centred proton reduction catalysis in a model of [NiFe] hydrogenase. Nat. Chem. 8, 1054-1060 (2016).

2. Ren, S. et al. Molecular electrocatalysts can mediate fast, selective $\mathrm{CO} 2$ reduction in a flow cell. Science (80-. ). 365, 367-369 (2019).

3. Nam, D. H. et al. Molecular enhancement of heterogeneous CO2 reduction. Nat. Mater. 19, 266276 (2020).

4. Rosser, T. E., Gross, M. A., Lai, Y. H. \& Reisner, E. Precious-metal free photoelectrochemical water splitting with immobilised molecular Ni and Fe redox catalysts. Chem. Sci. 7, 4024-4035 (2016).

5. Sun, L., Reddu, V., Fisher, A. C. \& Wang, X. Electrocatalytic reduction of carbon dioxide: Opportunities with heterogeneous molecular catalysts. Energy Environ. Sci. 13, 374-403 (2020).

6. Wang, M. et al. $\mathrm{CO} 2$ electrochemical catalytic reduction with a highly active cobalt phthalocyanine. Nat. Commun. 10, 1-8 (2019).

7. Zhang, B. \& Sun, L. Artificial photosynthesis: Opportunities and challenges of molecular catalysts. Chem. Soc. Rev. 48, 2216-2264 (2019).

8. Materna, K. L., Crabtree, R. H. \& Brudvig, G. W. Anchoring groups for photocatalytic water oxidation on metal oxide surfaces. Chem. Soc. Rev. 46, 6099-6110 (2017).

9. Hanna, C. M., Luu, A. \& Yang, J. Y. Proton-coupled electron transfer at anthraquinone modified indium tin oxide electrodes. ACS Appl. Energy Mater. 2, 59-65 (2019).

10. Creus, J. et al. A Million Turnover Molecular Anode for Catalytic Water Oxidation. Angew. Chemie - Int. Ed. 55, 15382-15386 (2016).

11. Ashford, D. L. et al. Water Oxidation by an Electropolymerized Catalyst on Derivatized Mesoporous Metal Oxide Electrodes. J. Am. Chem. Soc. 136, 6578-6581 (2014).

12. Liu, Y. \& McCrory, C. C. L. Modulating the mechanism of electrocatalytic CO 2 reduction by cobalt phthalocyanine through polymer coordination and encapsulation. Nat. Commun. 10, 1-10 (2019).

13. Krawicz, A. et al. Photofunctional construct that interfaces molecular cobalt-based catalysts for H2 production to a visible-light-absorbing semiconductor. J. Am. Chem. Soc. 135, 11861-11868 
(2013).

14. Schreiber, C. L. \& Smith, B. D. Molecular conjugation using non-covalent click chemistry. Nat. Rev. Chem. 3, 393-400 (2019).

15. Rojas, M. T., Kaifer, A. E., Königer, R. \& Stoddart, J. F. Supported Monolayers Containing Preformed Binding Sites. Synthesis and Interfacial Binding Properties of a Thiolated $\beta$-Cyclodextrin Derivative. J. Am. Chem. Soc. 117, 336-343 (1995).

16. Beulen, M. W. J. et al. Host-guest interactions at self-assembled monolayers of cyclodextrins on gold. Chem. - A Eur. J. 6, 1176-1183 (2000).

17. Beulen, M. W. J. et al. Self-assembled monolayers of heptapodant $\beta$-cyclodextrins on gold. Langmuir 14, 6424-6429 (1998).

18. Méndez-Ardoy, A., Steentjes, T., Kudernac, T. \& Huskens, J. Self-Assembled monolayers on gold of $\beta$-cyclodextrin adsorbates with different anchoring groups. Langmuir 30, 3467-3476 (2014).

19. Liu, W., Zhang, Y. \& Gao, X. Interfacial supramolecular self-assembled monolayers of C60 by thiolated $\beta$-cyclodextrin on gold surfaces via monoanionic C60. J. Am. Chem. Soc. 129, 4973-4980 (2007).

20. Freitag, M. \& Galoppini, E. Molecular host-guest complexes: Shielding of guests on semiconductor surfaces. Energy and Environmental Science 4, 2482-2494 (2011).

21. Freitag, M. \& Galoppini, E. Cucurbituril complexes of viologens bound to TiO2 films. Langmuir 26, 8262-8269 (2010).

22. Li, H. et al. Visible-Light-Driven Water Oxidation on a Photoanode by Supramolecular Assembly of Photosensitizer and Catalyst. Chempluschem 81, 1056-1059 (2016).

23. Kim, H. J., Lee, M. H., Mutihac, L., Vicens, J. \& Kim, J. S. Host-guest sensing by calixarenes on the surfaces. Chem. Soc. Rev. 41, 1173-1190 (2012).

24. Murray, J., Kim, K., Ogoshi, T., Yao, W. \& Gibb, B. C. The aqueous supramolecular chemistry of cucurbit[: N] urils, pillar [n] arenes and deep-cavity cavitands. Chem. Soc. Rev. 46, 2479-2496 (2017).

25. Méndez-Ardoy, A. et al. Electron-Transfer Rates in Host-Guest Assemblies at $\beta$-Cyclodextrin Monolayers. Langmuir 33, 8614-8623 (2017). 
26. Al-Soufi, W. et al. Fluorescence correlation spectroscopy, a tool to investigate supramolecular dynamics: Inclusion complexes of pyronines with cyclodextrin. J. Am. Chem. Soc. 127, 8775-8784 (2005).

27. Domi, Y., Yoshinaga, Y., Shimazu, K. \& Porter, M. D. Characterization and optimization of mixed Thiol-Derivatized ??-Cyclodextrin/Pentanethiol Monolayers with high-density guest-accessible cavities. Langmuir 25, 8094-8100 (2009).

28. Perl, A. et al. Gradient-driven motion of multivalent ligand molecules along a surface functionalized with multiple receptors. Nat. Chem. 3, 317-322 (2011).

29. Chang, B. Y., Hong, S. Y., Yoo, J. S. \& Park, S. M. Determination of electron transfer kinetic parameters by fourier transform electrochemical impedance spectroscopic analysis. J. Phys. Chem. B 110, 19386-19392 (2006).

30. Lee, J.-Y. \& Park, S.-M. Electrochemistry of Guest Molecules in Thiolated Cyclodextrin SelfAssembled Monolayers: An Implication for Size-Selective Sensors. J. Phys. Chem. B 102, 99409945 (1998).

31. Habibzadeh, F., Miller, S. L., Hamann, T. W. \& Smith, M. R. Homogeneous electrocatalytic oxidation of ammonia to N 2 under mild conditions. Proc. Natl. Acad. Sci. U. S. A. 116, 2849-2853 (2019).

32. Nakajima, K., Toda, H., Sakata, K. \& Nishibayashi, Y. Ruthenium-catalysed oxidative conversion of ammonia into dinitrogen. Nat. Chem. 11, 702-709 (2019).

33. Dunn, P. L., Johnson, S. I., Kaminsky, W. \& Bullock, R. M. Diversion of Catalytic C-N Bond Formation to Catalytic Oxidation of NH 3 through Modification of the Hydrogen Atom Abstractor. J. Am. Chem. Soc. jacs.9b13706 (2020). doi:10.1021/jacs.9b13706

34. Adli, N. M., Zhang, H., Mukherjee, S. \& Wu, G. Review-Ammonia Oxidation Electrocatalysis for Hydrogen Generation and Fuel Cells. J. Electrochem. Soc. 165, J3130-J3147 (2018).

35. Sévery, L., Siol, S. \& Tilley, S. Design of Molecular Water Oxidation Catalysts Stabilized by Ultrathin Inorganic Overlayers-Is Active Site Protection Necessary? Inorganics 6, 105 (2018).

36. Hamai, S. Inclusion of methyl 2-naphthalenecarboxylate and dimethyl 2,3-, 2,6-, and 2,7naphthalenedicarboxylates by cyclodextrins in aqueous solution. Bull. Chem. Soc. Jpn. 83, 14891500 (2010). 
37. Organero, J. A., Tormo, L. \& Douhal, A. Caging ultrafast proton transfer and twisting motion of 1hyroxyl-2-acetonapthone. Chem. Phys. Lett. 363, 409-414 (2002).

38. Hutter, J., Iannuzzi, M., Schiffmann, F. \& Vandevondele, J. Cp2k: Atomistic simulations of condensed matter systems. Wiley Interdiscip. Rev. Comput. Mol. Sci. 4, 15-25 (2014).

39. Jackson, M. N. et al. Strong Electronic Coupling of Molecular Sites to Graphitic Electrodes via Pyrazine Conjugation. J. Am. Chem. Soc. 140, 1004-1010 (2018).

40. Veerbeek, J., Méndez-Ardoy, A. \& Huskens, J. Electrochemistry of Redox-Active Guest Molecules at $\beta$-Cyclodextrin-Functionalized Silicon Electrodes. ChemElectroChem 4, 1470-1477 (2017).

41. Cherevko, S., Topalov, A. A., Zeradjanin, A. R., Katsounaros, I. \& Mayrhofer, K. J. J. Gold dissolution: Towards understanding of noble metal corrosion. RSC Adv. 3, 16516-16527 (2013).

42. Widrig, C. A., Chung, C. \& Porter, M. D. The electrochemical desorption of n-alkanethiol monolayers from polycrystalline Au and Ag electrodes. J. Electroanal. Chem. 310, 335-359 (1991).

43. Wong, E. H. J., May, G. L. \& Wilde, C. P. Oxidative desorption of thiols as a route to controlled formation of binary self assembled monolayer surfaces. Electrochim. Acta 109, 67-74 (2013).

44. Hashmi, A. S. K. \& Hutchings, G. J. Gold Catalysis. Angewandte Chemie - International Edition 45, 7896-7936 (2006).

45. Bangle, R., Sampaio, R. N., Troian-Gautier, L. \& Meyer, G. J. Surface Grafting of Ru(II) DiazoniumBased Sensitizers on Metal Oxides Enhances Alkaline Stability for Solar Energy Conversion. ACS Appl. Mater. Interfaces 10, 3121-3132 (2018).

46. Ide, A. et al. Monitoring bisphosphonate surface functionalization and acid stability of hierarchically porous titanium zirconium oxides. Langmuir 27, 12985-12995 (2011).

47. Biggs, C. I., Edmondson, S. \& Gibson, M. I. Thiol-ene immobilisation of carbohydrates onto glass slides as a simple alternative to gold-thiol monolayers, amines or lipid binding. Biomater. Sci. 3, 175-181 (2015).

48. Deskins, N. A., Rousseau, R. \& Dupuis, M. Localized electronic states from surface hydroxyls and polarons in TiO2(110). J. Phys. Chem. C 113, 14583-14586 (2009).

49. Adler, D. Electrical and optical properties of transition- metal oxides. Radiat. Eff. 4, 123-131 (1970). 
50. Wang, W. \& Kaifer, A. E. Transfer of cationic cucurbit[7]uril inclusion complexes from water to non-aqueous solvents. Supramol. Chem. 22, 710-716 (2010).

51. Connors, K. A. The Stability of Cyclodextrin Complexes in Solution. Chem. Rev. 97, 1325-1358 (2002). 